\title{
Genomic analysis reveals selection signatures of the Wannan Black pig during domestication and breeding
}

\author{
Wei Zhang ${ }^{1, a}$, Min Yang ${ }^{1, a}$, Yuanlang Wang ${ }^{1}$, Xudong Wu ${ }^{1}$, Xiaodong Zhang ${ }^{1}$, \\ Yueyun Ding ${ }^{1, *}$, and Zongjun Yin ${ }^{1, *}$
}

\author{
* Corresponding Authors: \\ Yueyun Ding \\ Tel: +86-13966675382, Fax: +86-0551-65787303, \\ E-mail: dingyueyun@ahau.edu.cn \\ Zongjun Yin \\ Tel: +86-0551-65787303, Fax: +86-0551-65787303, \\ E-mail: yinzongjun@ahau.edu.cn
}

'College of Animal Science and Technology, Anhui Agricultural University, Hefei 230036, China

a These authors contributed equally to this work.

ORCID

Wei Zhang

https://orcid.org/0000-0001-7673-1924

Min Yang

https://orcid.org/0000-0003-3350-0530

Yuanlang Wang

https://orcid.org/0000-0001-9245-2554

Xudong Wu

https://orcid.org/0000-0002-3077-9575

Xiaodong Zhang

https://orcid.org/0000-0003-4677-1620

Yueyun Ding

https://orcid.org/0000-0001-7559-0736

Zongjun Yin

https://orcid.org/0000-0001-9893-743X

Submitted Apr 7, 2019; Revised Jun 9, 2019;

Accepted Aug 1, 2019
Objective: The Wannan Black pig is a typical Chinese indigenous, disease-resistant pig breed with high fertility, and a crude-feed tolerance that has been bred by artificial selection in the south of Anhui province for a long time. However, genome variation, genetic relationships with other pig breeds, and domestication, remain poorly understood. Here, we focus on elucidating the genetic characteristics of the Wannan Black pig and identifying selection signatures during domestication and breeding.

Methods: We identified the whole-genome variation in the Wannan Black pig and performed population admixture analyses to determine genetic relationships with other domesticated pig breeds and wild boars. Then, we identified the selection signatures between the Wannan Black pig and Asian wild boars in 100-kb windows sliding in $10 \mathrm{~kb}$ steps by using two approaches: the fixation index $\left(\mathrm{F}_{\mathrm{ST}}\right)$ and $\pi$ ratios.

Results: Resequencing the Wannan Black pig genome yielded $501.52 \mathrm{G}$ of raw data. After calling single-nucleotide variants (SNVs) and insertions/deletions (InDels), we identified 21,316,754 SNVs and 5,067,206 InDels (2,898,582 inserts and 2,168,624 deletions). Additionally, we found genes associated with growth, immunity, and digestive functions.

Conclusion: Our findings help in explaining the unique genetic and phenotypic characteristics of Wannan Black pigs, which in turn can be informative for future breeding programs of Wannan Black pigs.

Keywords: Wannan Black Pig; Selection Signature; Genome Variation; Porcine Industry

\section{INTRODUCTION}

Since their domestication some 9,000 years ago [1], approximately 300 breeds of pig (Sus scrofa) have been bred globally under natural and artificial selection [2] that have resulted in a range of adaptations and phenotypic features, which now distinguish those breeds from their wild counterparts. With the development of genetics and genomic technologies, more genes and genomes are being revealed [3,4], and as the cost of sequencing has declined, powerful tools are now available that can be readily used for studying the evolution of species and targeted selection to elucidate the involvement of natural processes and human technology in the evolutionary process and how both have shaped modern animal genomes to provide novel insights for further improving livestock.

A novel beneficial variant that has been under selection pressure usually shows a high population frequency and long-range linkage disequilibrium [5]. Geneticists have proposed a series of methods based on the decay of linkage disequilibrium and variation of allele frequency to detect genes under selection [6-8]. Numerous studies, based on chip or sequencing data, have been carried out to detect genome-wide selective signatures in 
humans $[3,9]$ and in various agricultural species, including pigs $[4,10,11]$, cattle [12], dogs [13], goats and sheep [14], chicken [8], and ducks [15] and have revealed a series of genes associated with hair development, skin pigmentation, coat color, body size, fertility, horn, environmental adaptation, adaptation to a starch-rich diet, and disease-resistance. Combined calculations of fixation index $\left(\mathrm{F}_{\mathrm{ST}}\right)[6]$ and $\pi$ ratio [7] to detect selection signatures have been used in many studies [16].

The Wannan Black pig is a typical Chinese indigenous, disease-resistant breed with high fertility, and a crude-feed tolerance that has been bred in the south of Anhui province by artificial selection for a long time. Our previous study about retinol-binding protein 4 (RBP4) gene and cholesteryl estertransfer protein $(C E T P)$ gene in Wannan black pig revealed that RBP4 was significantly associated with average back-fat thickness and meat color $b^{*}$ value, and identified a mutation in CETP, which had significant effects on the expression in liver and correlated positively with serum lipid and meat fat phenotypes [17,18]. Moreover, other genes and microRNAs related with immune traits and fertility have been identified $[19,20]$. However, these studies were performed only on a few genes and microRNAs. Further research to identify more genes that might assist us in elucidating the special characteristics of the Wannan Black pig breed, is required. Therefore, it is necessary to investigate selection signatures in Wannan Black pig to uncover its genetic characters.

Here, we focused on Wannan Black pig domestication and breeding using a resequencing dataset of 20 unrelated Wannan Black pigs and 28 other wild and domesticated pigs to find potential genomic evidence linking the domestication of Wannan Black pig with their breed characteristics on the basis of $\mathrm{F}_{\mathrm{ST}}$ and $\pi$ ratio. The findings herein will provide new insights to expand our understanding of the genetic base that determines the unique traits of the Wannan Black pig.

\section{MATERIALS AND METHODS}

Experimental animals and whole-genome sequencing All experimental procedures were carried out in strict accordance with the protocols approved by the Anhui Agricultural University Animal Ethics Committee under permission No. AHAU20140215. Ear tissue was collected from 20 Wannan Black pigs (10 females and 10 males) from the Wannan Black pig conservation farm (Anhui, China) for high-throughput resequencing. DNA samples were extracted from all pigs using the Qiagen DNeasy Tissue kit (Qiagen, Dusseldorf, Germany), and the integrity and purity of the DNA were verified by agarose gel electrophoresis and A260/280 ratio. The genomic DNA was then processed with the Covaris system end-repair, A-tailing, ligation of pair-ended adapter, size-selection for sequencing and amplification. Finally, amplified fragments were sequenced on a HiSeqX platform using the protocols recommended by the manufacturer at Novogene (Beijing, China).

Additional resequencing data from 28 individuals were downloaded from NCBI (https://www.ncbi.nlm.nih.gov/), including 13 Asian wild boars, three European wild boars, and one of each of the following breeds: Yorkshire, Landrace, Bamaxiang, Rongchang, Meishan, Tibetan, Laiwu, Hetao, Sus barbatus, Sus cebifrons, Sus verrucosus, and Phacochoerus africanus (Supplementary Table S1).

Reads alignment, variant calling, and annotation To avoid low-quality reads, raw data were processed using the NGSQC Toolkit (v.2.30) [21]. Firstly, reads with an adapter sequence were deleted; next, reads containing more than $10 \%$ $\mathrm{N}$ bases were removed, and reads containing more than $50 \%$ low-quality bases (quality value $\leq 5$ ) were discarded. Thus, only paired reads were preserved. The filtered pair-ended reads were aligned to the Sus scrofa reference genome (Suss11.1, GenBank assembly GCF_000003025.6) using BWA-MEM [22], and the bam file was sorted using Picard SortSam and improved using Picard MarkDuplicates (http://picard.source forge.net). Next, we used SAMtools mpileup [23] and GATK HaplotypeCaller algorithm [24] to call variants of the Wannan Black pig. Integrated variants from two algorithms were filtered using the tool "VariantFiltration" of GATK by "Qual ByDepth $<20.0$; ReadPosRankSum $<-8.0$; FisherStrand $>10.0$; Quality $<$ MEANQUAL", and then we recalibrated the bam file. Finally, the variants were called through GATK with "HaplotypeCaller" "GenotypeGVCFs" and "SelectVariants" parameter. To filter variants and avoid possible false positives, the tool "VariantFiltration" of GATK was used with the following options: "Depth <4.0; DP >1,000.0; RMS mapping quality $<20.00$ " and "-cluster 2 -window 4" for singlenucleotide variants (SNVs); "QD <2.0; FS > 200.0; ReadPos RankSum <-20.0" for insertions/deletions (InDels). After filtering, the variants were annotated with the ANNOVAR v2013-08-23 software [25]. This process of data filtering and variant calling was applied to sequencing data from Wannan pigs and other domesticated breeds and wild boars.

\section{Phylogenic construction, principle component analysis, and admixture analysis}

To infer the population structure, we downloaded the sequencing data of 28 individuals from different geographical locations (Figure 1). To estimate the genetic and population structure of the pigs in our study, we filtered all autosome SNVs with a minor allele frequency (MAF) $<0.05$ and linkage disequilibrium $\left(\mathrm{r}^{2}\right)<0.2$ between studied pig populations, site missing rate $<0.05$, and quality value $<30$, and then we converted the filtered VCF file to PLINK input file formats (.map and .ped). First, we performed principle component 


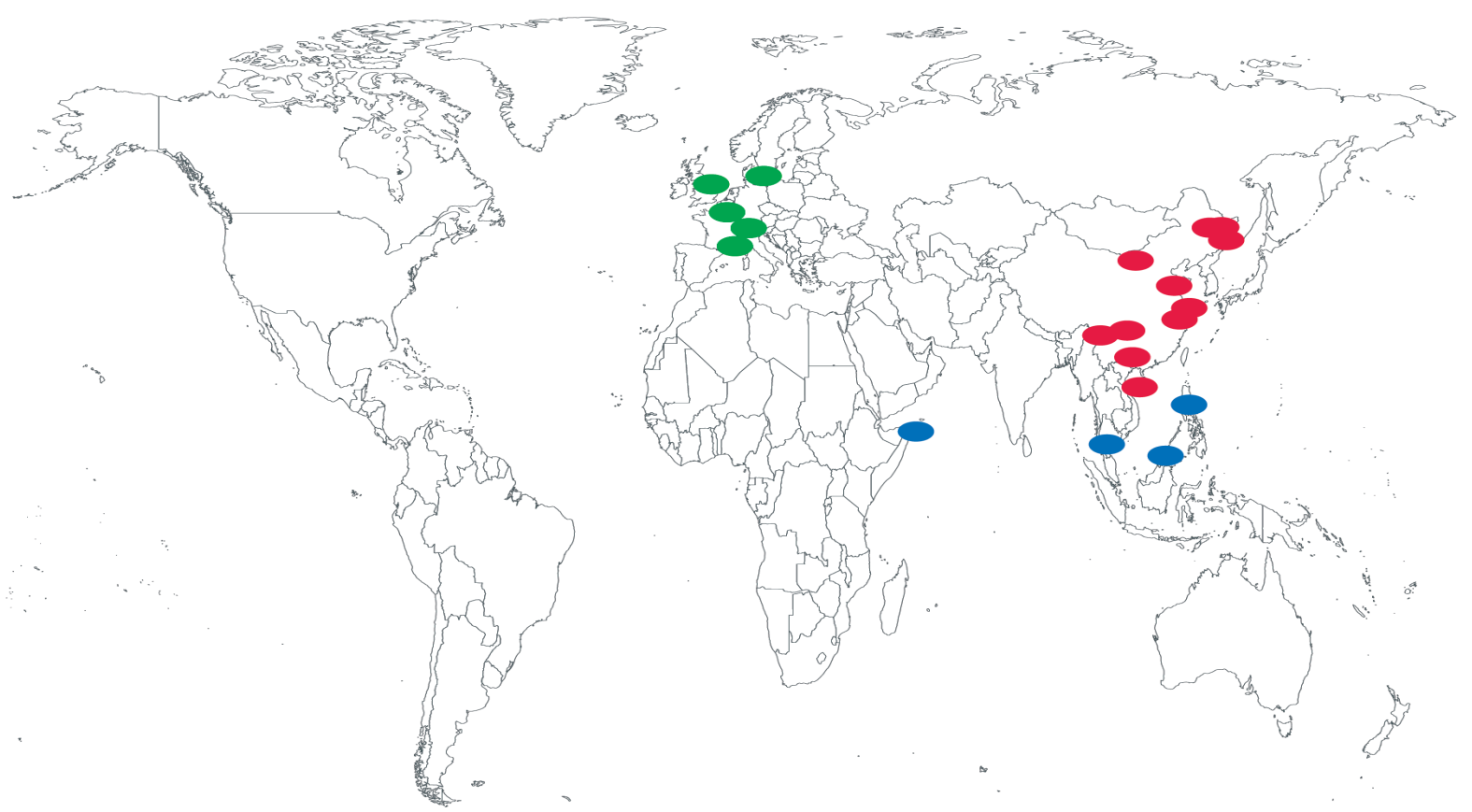

Figure 1. Geographic origin of the 19 analyzed pig breeds, including Europe and America ( $n=5$; green circles), Africa and Sus ( $n=4$; blue circle), Asia ( $n=10$; red circles).

analysis (PCA) with map and ped files using the GCTA software (v.1.25) using GCTA [26] "--make-grm” and "--grm tmp --pca 3” to generate .eigenval and .eigenvec files. Secondly, we performed a population admixture analysis using the ADMIXTURE software (v.1.3) [27] to infer the true number of genetic populations (clusters or K) among the pig breeds. Prior population information was ignored before testing and identifying distinct genetic populations and assigning individuals to populations. ADMIXTURE uses cross validation procedures to estimate the most preferable $K$-value, which is considered to be the one that exhibits a low crossvalidation error, compared to other $K$-values and is considered to be the most probable number of inferred populations. Lastly, we performed a phylogenetic tree analysis by generating an identical-by-state distance matrix using the PLINK software v.1.90 (PLINK, RRID: SCR_001757) [28]. We then constructed neighbor-joining trees using SNPHhylo (v.2014 0701) [29]; trees were drawn using FigTree (v.1.4.0) [30].

\section{Detection of genome-wide selective sweeps}

Regions under selection between the Wannan Black pig and the Asian wild boar were identified based on two different statistics, i.e. $\mathrm{F}_{\mathrm{ST}}$ and $\pi$ ratio. Prior to analysis, a series of qualitycontrol procedures were implemented using VCFtools to achieve high-quality data by removing any SNVs with call rates $<0.90$ and with MAF $<0.05$ and by excluding individuals with a max missing count $>4$. Following quality control, missing genotypes were assigned using the BEAGLE software [31]. A $100-k b$ sliding window approach with $10-k b$ step-size was applied to calculate these statistics with PopGenome [32]. To define putative genomic regions under selection, first we defined the selection region of the two approaches based on 0.01 and 0.05 level. Then, the overlapped regions corresponding to 0.01 and 0.05 level of the two approaches were defined as the final selection signatures.

To explore the potential biological significance of genes within these sweep regions, gene ontology (GO) and Kyoto encyclopedia of genes and genomes (KEGG) analyses were carried out using Database for Annotation, Visualization and Integrated Discovery (DAVID, v.6.8) [33]. Benjamini-Hochberg false discovery rates [34] were used for correcting $\mathrm{p}$ values. Only terms with a $\mathrm{p}<0.05$ were considered to be significant.

\section{RESULTS}

\section{Genome resequencing and genetic variation}

Resequencing of the Wannan Black pig $(n=20$, Figure 2) yielded $501.52 \mathrm{G}$ of raw data. After mapping these to the Sus scrofa reference genome 11.1, an average of $84.74 \%$ ( $83.44 \%$ $85.66 \%$ ) of reads were mapped. The depth of the experimental population ranged from 7.10 to 11.38 folds with an average of 9.43. The depth of at least one or four of the experimental population averaged 98.02 and 89.31 , respectively (Table 1 ). The data have been submitted to NCBI with accession number PRJNA524263.

After calling SNVs and InDels, we identified 21,316,754 SNVs and 5,067,206 InDels (2,898,582 inserts and 2,168,624 deletions). Among the SNVs, 3,896,353 were newly identi- 


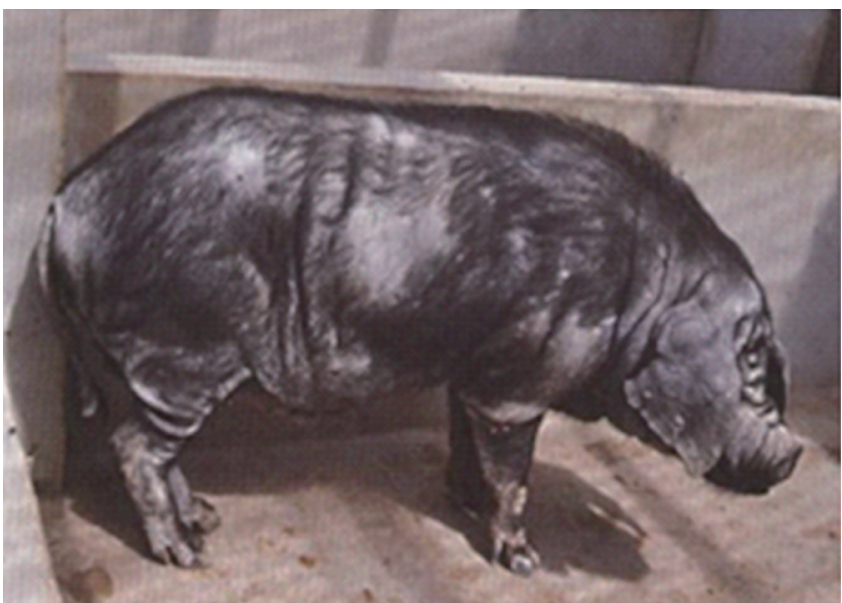

Figure 2. The Wannan Black pig.

fied and were not included in the dbSNP database (ftp://ftp. ncbi.nih.gov/snp/organisms/pig 9823/VCF/). Of all identified SNVs, intergenic variants were the most abundant (59.6\%) (Table 2). Meanwhile, 144,815 were synonymous, 64,671 were non-synonymous, and 657 were stop-gain variants (Table 2). Additionally, we observed that the average ratio of transitions/ transversions was 2.28. Of all identified InDels, most variants were in intergenic regions (59.5\%). There were 13,766 in coding domains; meanwhile, 10,302 were frameshift variants, of which 6,447 were frameshift insertions and 3,855 were frameshift deletions (Table 2).
Table 2. Summary of identified single nucleotide variants and insertions/ deletions

\begin{tabular}{|c|c|}
\hline Variant type & No. of variants \\
\hline SNV & $21,316,754$ \\
\hline Intergenic & $12,722,683$ \\
\hline Intragenic & $8,347,223$ \\
\hline Downstream & 124,895 \\
\hline Upstream & 121,953 \\
\hline Splicing site & 1198 \\
\hline 5' UTR & 44,897 \\
\hline 3' UTR & 198,836 \\
\hline Intron & $7,892,061$ \\
\hline Coding domain & 210,231 \\
\hline Synonymous & 144,815 \\
\hline Non-synonymous & 64,671 \\
\hline InDel & $5,067,206$ \\
\hline Intergenic & $3,014,421$ \\
\hline Intragenic & $1,989,666$ \\
\hline Downstream & 32,210 \\
\hline Upstream & 30,909 \\
\hline Splicing site & 3840 \\
\hline 5' UTR & 10,002 \\
\hline 3' UTR & 50,007 \\
\hline Intron & $1,912,051$ \\
\hline Coding domain & 13,766 \\
\hline Frameshift deletion & 3,855 \\
\hline Frameshift insertion & 6,447 \\
\hline Non-frameshift deletion & 1,520 \\
\hline Non-frameshift insertion & 1,641 \\
\hline
\end{tabular}

SNVs, single nucleotide variants; InDels, insertions/deletions; UTR, untranslated region.

Table 1. Summary statistics of whole-genome sequencing

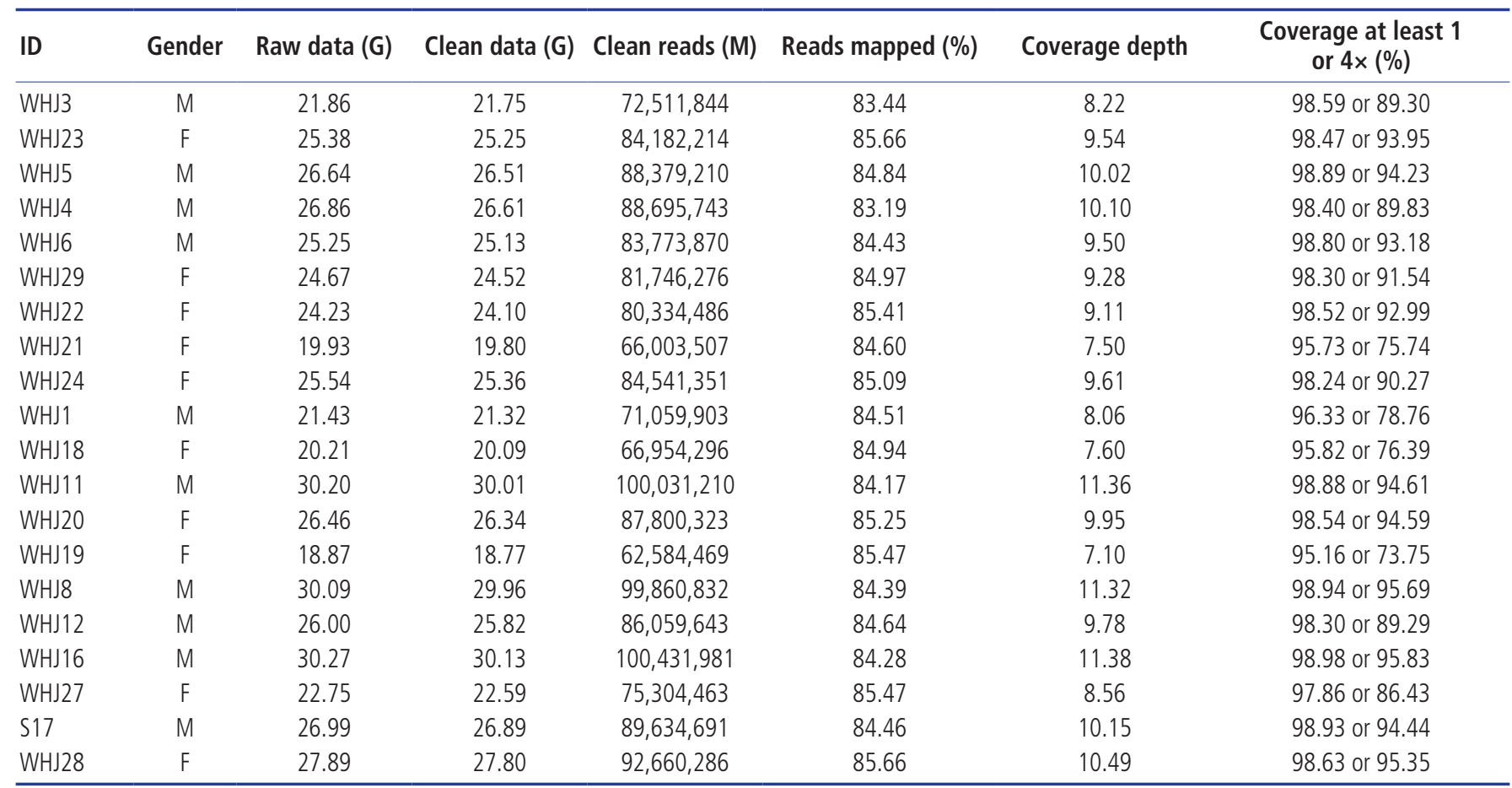


Phylogenic construction, principle component analysis and admixture analyses

After quality control, 903,292 variants were used to construct the neighbor-joining tree and run PCA and admixture analysis. To assess the phylogenetic relationship among the pig breeds in this study, unrooted phylogenetic trees were con-

(A)

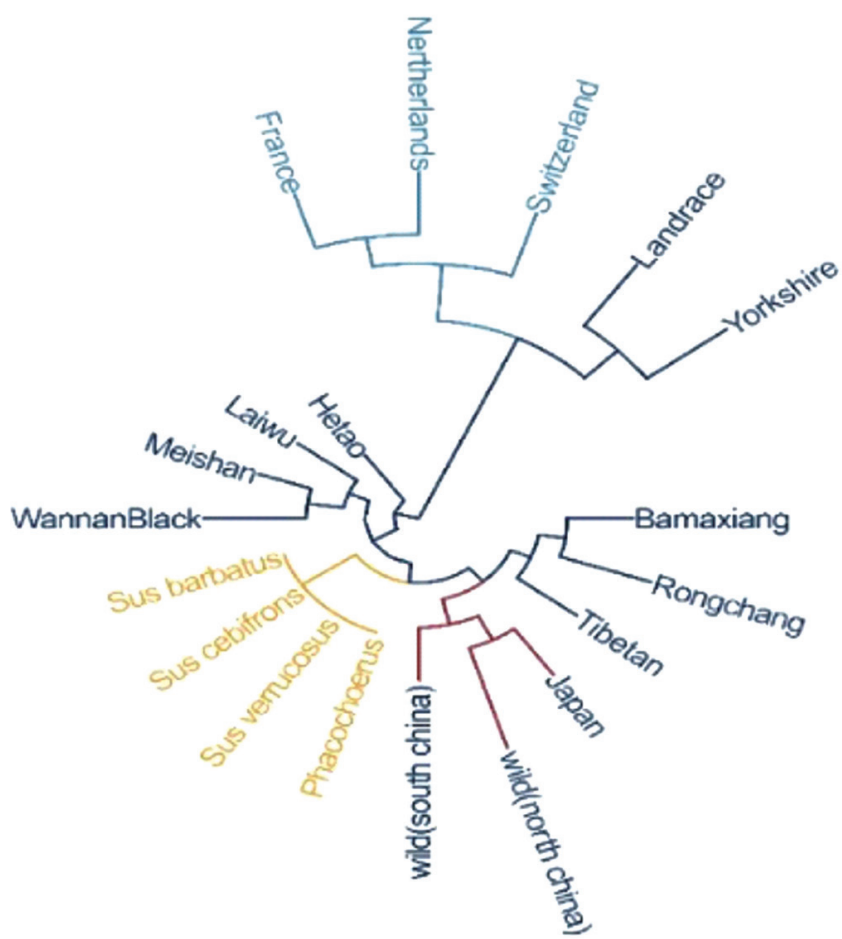

(C)

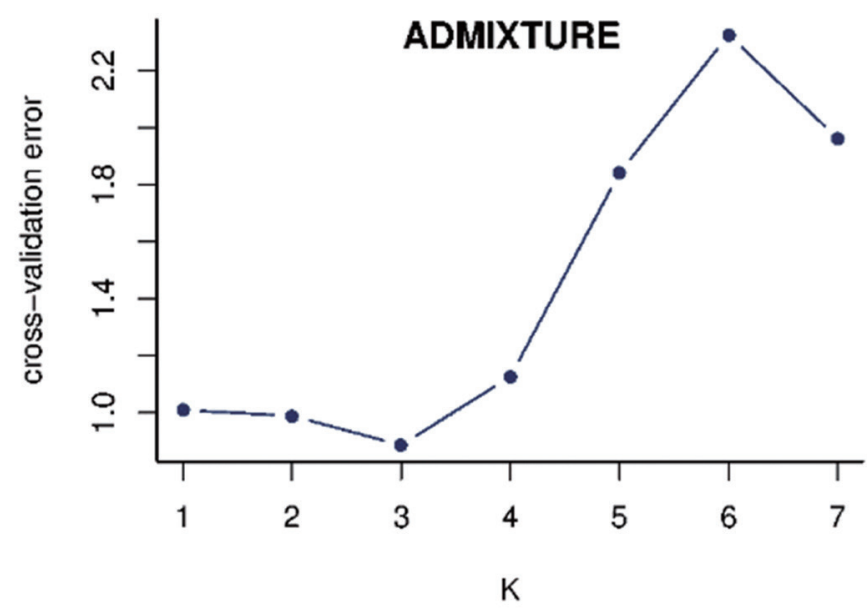

structed from the variants after filtering (Figure $3 \mathrm{~A}$ ). The branches of the phylogenetic tree were grouped as expected and were consistent with the results of PCA (Figure 3B), thus revealing strong clustering into three distinct genetic groups comprising Asian wild and domesticated boar, European wild and domesticated pigs, and the other four breeds. To infer
(B)

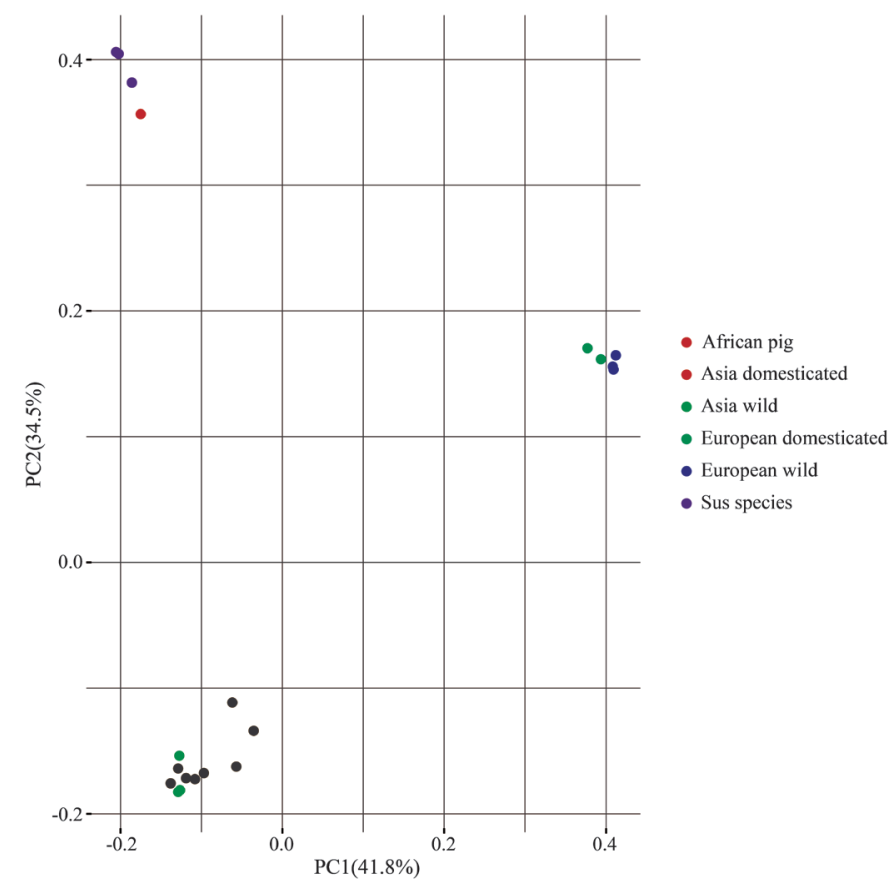

(D)

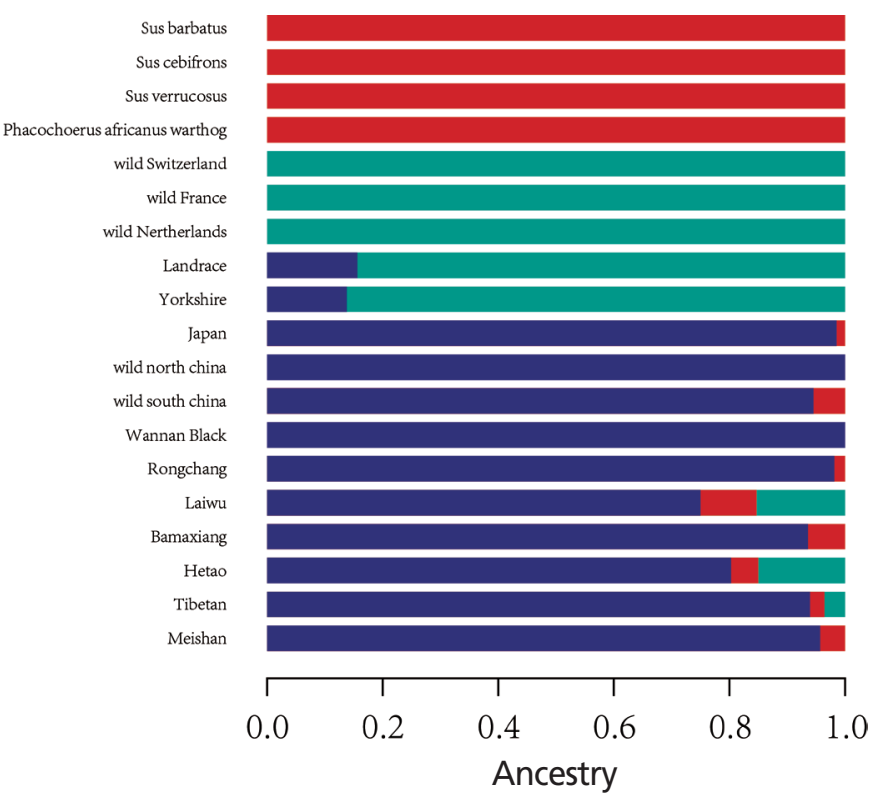

Figure 3. Population genetic structure of pig populations. (A) Neighbor-joining tree constructed from single-nucleotide variants (SNVs) data among 19 subspecies. (B) Principle component analysis plot of pig populations. Different colors represent different subspecies. (C) Cross-validation errors for diverse $k$ values. (D) Population structure of study population. The length of different colors represents proportions of ancestry from ancestral populations; breed names are indicated on the left. 
population admixture, we chose the lowest cross-validation error value $(k=3)$, which was taken as the most probable number of inferred populations (Figure 3C). Three clusters were observed: Phacochoerus africanus and Sus species, European wild and domesticated pigs, and Asian wild and domesticated pigs. The results of admixture analysis are shown in Figure 3D with a similar cluster pattern to those found in the PCA plots.

(A)

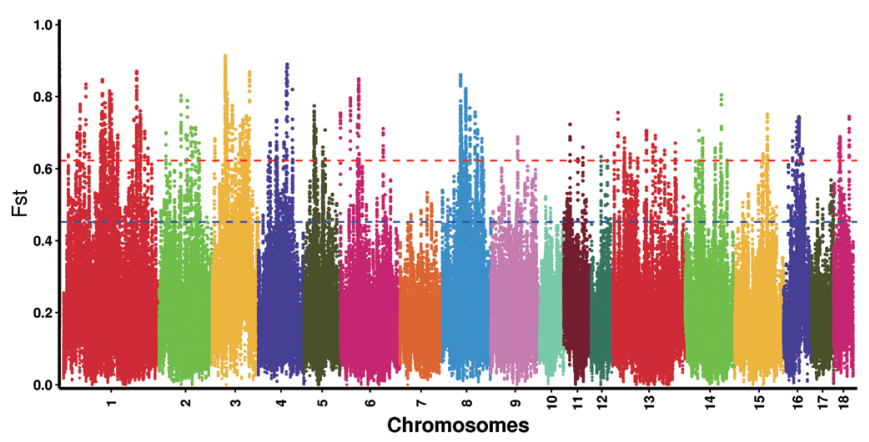

Selection detected by $\mathrm{F}_{\mathrm{ST}}$ and $\pi$ ratio

After quality control of the SNVs used for selection signature identification, there were 17,067,382 variants. Of these, $16,510,132$ were shared with Asian wild boars. The average nucleotide diversity was 0.00213 and 0.00227 for Wannan Black pigs and Asian wild boars, respectively. The genome distribution of the two statistics is shown in Figure 4A and 4B. Twenty-eight selected regions were identified as having extremely high $\mathrm{F}_{\mathrm{ST}}$ values (1\%) and significantly high $\pi$ ratios

(B)

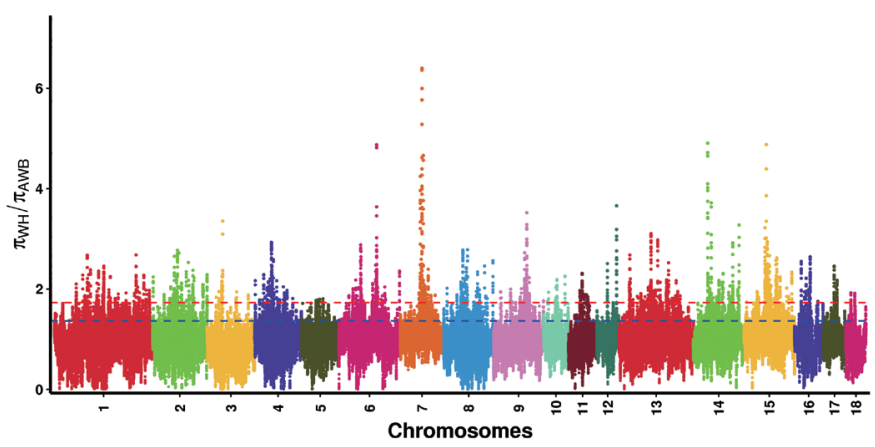

(C)

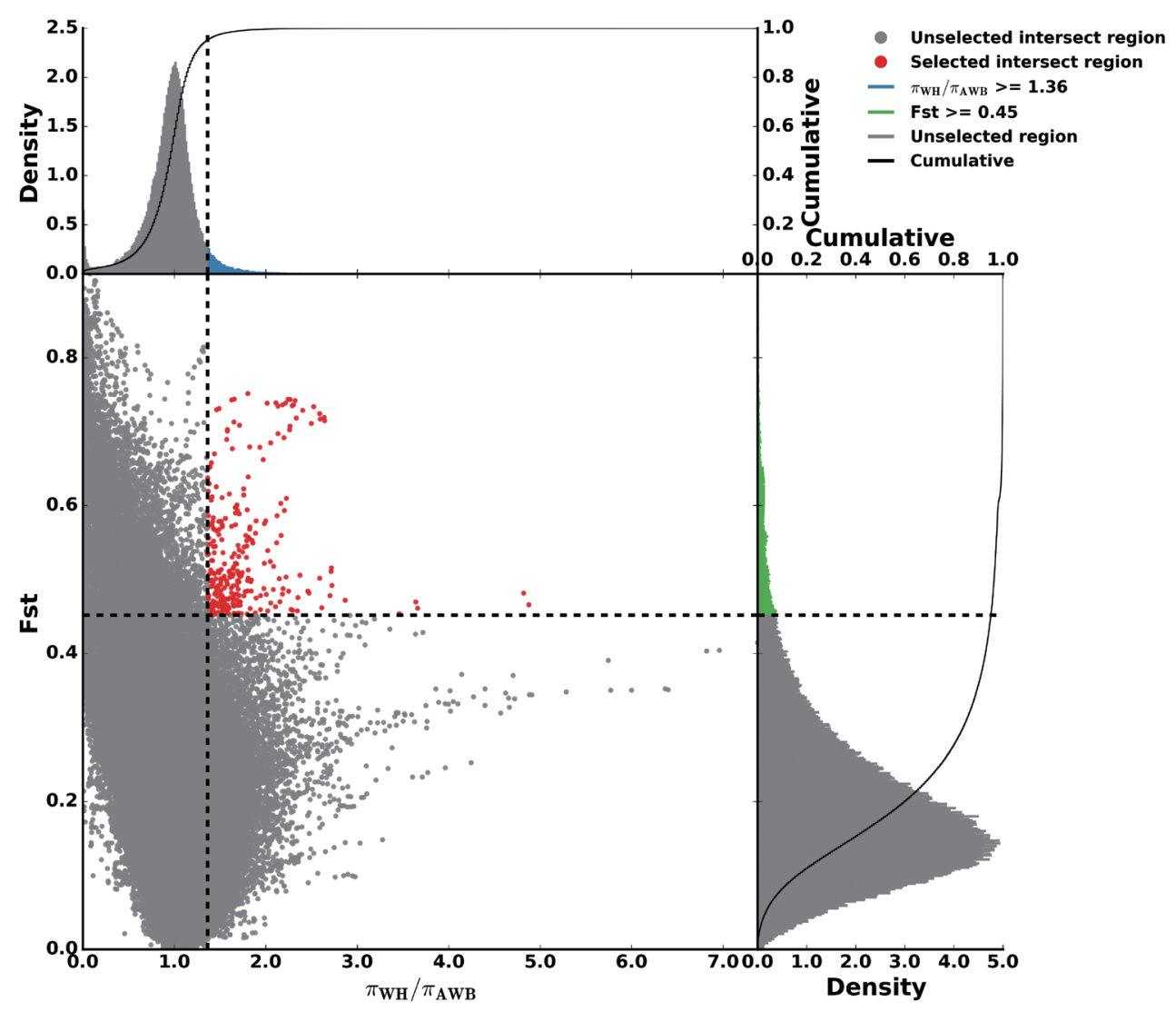

Figure 4. Distribution of $F_{S T}$ values and $\pi$ ratios calculated in $100 \mathrm{~kb}$ windows with $10 \mathrm{~kb}$ steps. (A) Distribution of $\mathrm{F}_{\mathrm{ST}}$ values among autosome chromosomes. The red line represents the 0.01 level, and the blue line represents the 0.05 level. (B) Distribution of $\pi$ ratios among autosomal chromosomes. (C) Intersection of the two methods used to identify high-quality selection regions. Data points located to the right of vertical dashed line (corresponding to the $5 \%$ right tails of the empirical $\theta \pi$ ratio distribution), and above the horizontal dashed line (the $5 \%$ right tail of the empirical $F_{S T}$ distribution) were identified as selected regions for Wannan black pig (red points). 
(1\%) (Supplementary Table S2). Five genes were identified within the regions (Supplementary Table S3); 286 selective regions (threshold, $5 \%$; $\mathrm{F}_{\mathrm{ST}}, 0.45 ; \pi$ ratio, 1.36 ) were identified in Wannan Black pigs (Figure 4C, Supplementary Table S4), which harbored 105 genes (Supplementary Table S5). For further analysis of the genes identified by DAVID, 41 GO terms were identified (Supplementary Table S6). The clusters were related to "reproductive process" "immune system process" "response to stimulus" and "growth" (Figure 5A). For KEGG analysis, 44 pathways were enriched, most of which were related to the immune system, signal transduction, and environmental adaptation, such as "T-cell receptor signaling

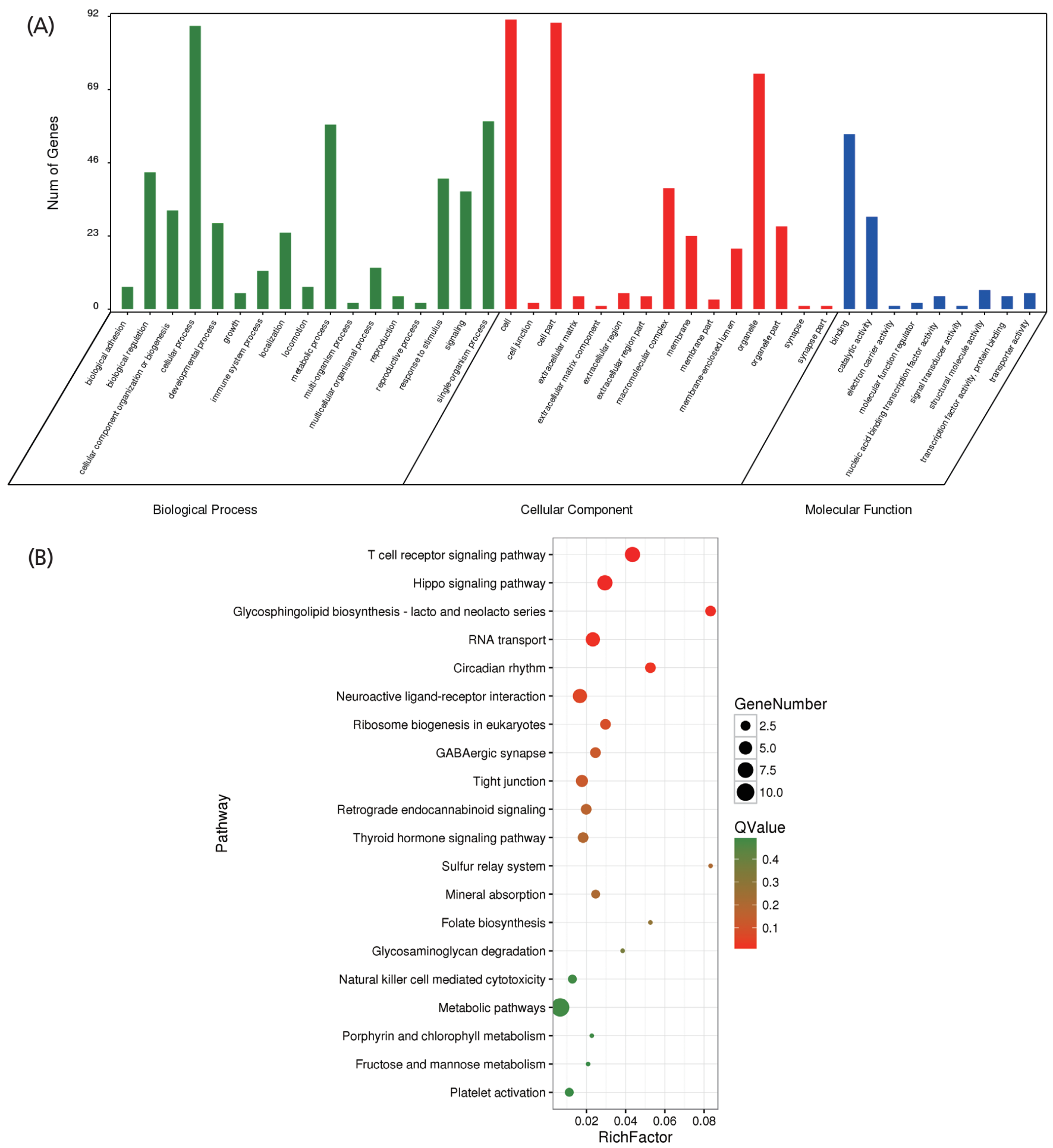

Figure 5. Results of the gene ontology (GO) and Kyoto encyclopedia of genes and genomes (KEGG) analyses for the genes harbored in the selection regions. (A) GO terms of the identified genes. (B) Top 20 enrichment pathways. 
pathway" "hippo signaling pathway" "Circadian rhythm" and "RNA transport" (Figure 5B, Supplementary Table S7).

\section{DISCUSSION}

Global meat production relies heavily on the capacity and effectiveness of pig breeding. To better understand the genetics underlying their domestication, we performed whole-genome sequencing on 20 Wannan Black pigs and downloaded sequencing data of 28 individuals. To our knowledge, this is the first study to characterize the genetic variation, phylogenetic relationships, population structure, and domestication of the Wannan Black pig. We observed $21 \mathrm{M} \mathrm{SNVs}$ and $3 \mathrm{M}$ InDels in the Wannan Black pig genome and a low nucleotide diversity, compared to Asian wild boars.

To reveal the selection signatures of the Wannan Black pig during domestication and breeding, we first selected regions within the top $1 \%$ of $\mathrm{F}_{\mathrm{ST}}$ and $\pi$ ratios and found five protein coding genes: peptidylprolyl isomerase domain and WD repeat containing 1 , which is one of the three classes of peptidylprolyl isomerases found in all eukaryotic and prokaryotic organisms, and viruses, assisting in protein folding [35]; ADAM metallopeptidase with thrombospondin type 1 motif 6 , which has been found to be associated with cardiac conduction [36]; tripartite motif containing 23 , whose function remains largely unknown; collagen type $\mathrm{V}$ alpha 2 chain, which has been shown to be associated with aortic aneurysms and dissections [37]; and centromere protein $\mathrm{K}$, which has been shown to be associated with catastrophic chromosome segregation defects [38]. GO and KEGG analysis of six genes showed significant association with the digestive system and metabolism, which to some extent may indicate their involvement in the crude-feed tolerance of the Wannan Black pig breed.

When we relaxed the threshold from $1 \%$ to $5 \%$, a total of 105 genes were identified in 286 selective regions. The genes were significantly enriched in terms of the immune system, environmental adaption, and signal transduction, such as "T-cell receptor signaling pathway" (seven genes), "hippo signaling pathway" (seven genes), "digestive system" (mineral absorption, two genes) "glycosphingolipid biosynthesislacto and neolacto series" (three genes), "RNA transport" (six genes)", "circadian rhythm" (three genes). The T-cell receptor signaling pathway can regulate generic and specialized functions, leading to T-cell proliferation, and cytokine production and differentiation into cells [39]. Hippo signaling is an evolutionarily-conserved signaling pathway that controls organ size in a variety of organisms from flies to humans [40]. The mineral absorption pathway can regulate intestinal calcium transport [41].

The GO analysis identified "reproductive process" (two genes), "immune system process" (12 genes), "growth" (five genes), "response to stimulus" (41 genes), and "molecular function regulator" (two genes). Of the two genes found for this process, Bardet-Biedl syndrome 4 can directly affect the proliferation and differentiation of adipocytes [42]. The immune system process is an organismal system for calibrated responses to potential internal or invasive threats. Of the 12 genes associated with the immune system, zinc finger protein 366 encodes the RNA-binding tristetraprolin, which is needed for $\mathrm{CD}^{+}$T-cell production of interferon- $\gamma$ (IFN- $\gamma$ ) in vivo. IFN $-\gamma$ produces cytotoxic $\mathrm{T}$ lymphocytes that are essential for host defense against viral infection and cancer [43]. Polymorphisms at the tumor necrosis factor (TNF) superfamily member 15 locus, which encodes the TNF superfamily cytokine commonly known as tumor necrosis factor-like ligand $1 \mathrm{~A}$, are associated with susceptibility to inflammatory bowel disease in a range of people [44]. A SH2 domain containing $1 \mathrm{~A}$ gene mutation in pediatric patients can regulate B-cell lymphoma [45]. The PAF1 homolog, Paf1/RNA polymerase II complex component gene can regulate RNA polymerase II (Pol2) movement through chromatin and the co-transcriptional processing and fate of nascent transcripts [46]. Macrophage stimulating 1 can regulate post-infarction cardiac injury through the JNK-Drp1-mitochondrial fission pathway [47]. Our previous research on MicroRNA-21 and microRNA-214 revealed that they play an important role in the regulation of estrous during porcine reproduction [20]. Analysis of the natural resistance-associated macrophage protein 1 encoding gene in Wannan black pig and Yorkshire identified a single nucleotide polymorphism, which was significantly associated with level of white blood cell \% ( $\mathrm{p}=$ $0.04)$, monocyte $\%(\mathrm{p}=0.024)$, rate of cyotoxin in monocyte $\%(\mathrm{p}=0.013)$ and $\mathrm{CD}^{-} \mathrm{CD}^{+} \mathrm{T}$ lymphocyte $(\mathrm{p}=0.023)$ elucidating the disease-resistance of Wannan black pig compared to Yorkshire [19]. Our previous and whole-genome resequencing results suggest that the genes detailed above played crucial roles in Wannan Black pig domestication by altering the functional regulation of the immune system, environmental adaption, fertility, and the digestive system.

Glycan biosynthesis- and metabolism-related genes have also been under selection pressure in Wannan Black pigs. ST3 beta-galactoside alpha-2,3-sialyltransferase 6 plays a key role in selectin ligand synthesis in humans through the generation of functional sialyl Lewis X. In MRC IX patients, high expression of this gene is associated with lesser overall survival [48].

Growth-related genes were also found to be under strong selection in Wannan Black pigs. Bovine dilated cardiomyopathy (DCM) is an autosomal recessive genetic disorder causing congestive heart failure and subsequent death. Recently, a nonsense mutation, c.343C $>\mathrm{T}$, in the bovine optic atrophy 3 gene reportedly caused DCM in Holstein cattle in Switzerland [49]. A de novo mutation in transducin $\beta$-like $1 \mathrm{X}$-linked receptor 1 was found to be associated with autism 
spectrum disorder and epilepsy by altering Wnt/ $\beta$-catenin signaling activity [50].

\section{CONFLICT OF INTEREST}

We certify that there is no conflict of interest with any financial organization regarding the material discussed in the manuscript.

\section{ACKNOWLEDGMENTS}

This research was supported by grants from the National Natural Science Foundation of China (31572377), the National Sci-Tech Support Plan of China (No. 2015BAD03B01), the National Key Research and Development Program of China (No. 2017YFD0600805), and the Anhui Provincial Natural Science Foundation (No. 1508085QC52). We thank Editage for professional English language editing of the manuscript to ensure correct use of language and scientific style.

\section{REFERENCES}

1. Giuffra E, Kijas JMH, Amarger V, Carlborg Ö, Jeon JT, Andersson L. The origin of the domestic pig: independent domestication and subsequent introgression. Genetics 2000; 154:1785-91.

2. Veirano Fréchou R. The state of the world's animal genetic resources for food and agriculture. Acta Paediatr 2007;81: 21-4.

3. Enard D, Messer PW, Petrov D. Genome-wide signals of positive selection in human evolution. Genome Res 2014;24: 885-95.

4. Frantz LAF, Schraiber JG, Madsen O, et al. Evidence of longterm gene flow and selection during domestication from analyses of Eurasian wild and domestic pig genomes. Nat Genet 2015;47:1141-8. https://doi.org/10.1038/ng.3394

5. Grossman SR, Shlyakhter I, Karlsson EK, et al. A composite of multiple signals distinguishes causal variants in regions of positive selection. Science 2010;327:883-6. https://doi.org/10. 1126/science. 1183863

6. Lewontin RC, Krakauer J. Distribution of gene frequency as a test of the theory of the selective neutrality of polymorphisms. Genetics 1973;74:175-95.

7. Tajima F. Evolutionary relationship of DNA sequences in finite populations. Genet 1983;105:437-60.

8. Rubin C, Zody MC, Eriksson J, et al. Whole-genome resequencing reveals loci under selection during chicken domestication. Nature 2010;464:587-91. https://doi.org/10.1038/ nature 08832

9. Salas A. The natural selection that shapes our genomes. Forensic Sci Int Genet 2019;39:57-60. https://doi.org/10.1016/j.fsigen. 2018.12.003
10.Zhao P, Yu Y, Feng W, et al. Evidence of evolutionary history and selective sweeps in the genome of Meishan pig reveals its genetic and phenotypic characterization. GigaScience 2018; 7:giy058. https://doi.org/10.1093/gigascience/giy058

11.Rubin C, Megens H, Barrio AM, et al. Strong signatures of selection in the domestic pig genome. Proc Nat Acad Sci USA 2012;109:19529-36. https://doi.org/10.1073/pnas.1217149109

12. Kim J, Hanotte O, Mwai OA, et al. The genome landscape of indigenous African cattle. Genome Biol 2017;18:34. https:// doi.org/10.1186/s13059-017-1153-y

13. Axelsson E, Ratnakumar A, Arendt M, et al. The genomic signature of dog domestication reveals adaptation to a starchrich diet. Nature 2013;495:360-4. https://doi.org/10.1038/ nature11837

14. Alberto FJ, Boyer F, Orozco-terWengel P, et al. Convergent genomic signatures of domestication in sheep and goats. Nat Commun 2018;9:813. https://doi.org/10.1038/s41467-01803206-y

15.Zhang Z, Jia Y, Almeida P, et al. Whole-genome resequencing reveals signatures of selection and timing of duck domestication. GigaScience 2018;7:giy027. https://doi.org/10.1093/ gigascience/giy 027

16.Li M, Tian S, Jin L, et al. Genomic analyses identify distinct patterns of selection in domesticated pigs and Tibetan wild boars. Nat Genet 2013;45:1431-8. https://doi.org/10.1038/ng. 2811

17.Zhang X, Huang L, Wu T, Feng Y, Ding Y, Yin ZJ. Polymorphism of the retinol-binding protein 4 gene (RBP4) and its association with carcass and meat quality traits in swine. Turk J Vet Anim Sci 2015;39:395-400. https://doi.org/10.3906/vet-150257

18. Ding YY, Zhang W, Zhang MQ, et al. Functional and association studies of the cholesteryl ester transfer protein (CETP) gene in a Wannan Black pig model. Anim Genet 2015;46:7026. https://doi.org/10.1111/age.12370

19.Ding X, Zhang X, Yang Y, et al. Polymorphism, expression of natural resistance-associated macrophage protein 1 encoding gene (NRAMP1) and its association with immune traits in pigs. Asian-Australas J Anim Sci 2014;27:1189-95. https://doi. org/10.5713/ajas.2014.14017

20.Tian M, Zhang X, Ye PF, et al. MicroRNA-21 and microRNA-214 play important role in reproduction regulation during porcine estrous. Anim Sci J 2018;89:1398-405. https://doi.org/10.1111/ asj. 13087

21.Patel RK, Jain M. NGS QC Toolkit: A toolkit for quality control of next generation sequencing data. PLoS One 2012;7:e30619. https://doi.org/10.1371/journal.pone.0030619

22.Li H, Durbin R. Fast and accurate short read alignment with Burrows-Wheeler transform. Bioinformatics 2009;25:175460. https://doi.org/10.1093/bioinformatics/btp324

23.Li H, Handsaker B, Wysoker A, et al. The Sequence alignment/ map format and SAMtools. Bioinformatics 2009;25:2078-99. 
https://doi.org/10.1093/bioinformatics/btp352

24. McKenna A, Hanna M, Banks E, et al. The genome analysis toolkit: a MapReduce framework for analyzing next-generation DNA sequencing data. Genome Res 2010;20:1297-303. https://doi.org/10.1101/gr.107524.110

25. Wang K, Li M, Hakonarson H. ANNOVAR: functional annotation of genetic variants from high-throughput sequencing data. Nucleic Acids Res 2010;38:e164. https://doi.org/10.1093/ nar/gkq603

26. Yang J, Lee SH, Goddard ME, Visscher PM. GCTA: A tool for genome-wide complex trait analysis. Am J Hum Genet 2011;88:76-82. https://doi.org/10.1016/j.ajhg.2010.11.011

27. Holsinger KE, Weir BS. Genetics in geographically structured populations: defining, estimating and interpreting $\mathrm{F}_{\mathrm{ST}}$. Nat Rev Genet 2009;10:639-50. https://doi.org/10.1038/nrg2611

28. Purcell S, Neale B, Todd-Brown K, et al. PLINK: A tool set for whole-genome association and population-based linkage analyses. Am J Hum Genet 2007;81:559-75. https:/doi.org/ $10.1086 / 519795$

29.Lee TH, Guo H, Wang X, Kim C, Paterson AH. SNPhylo: A pipeline to construct a phylogenetic tree from huge SNP data. BMC Genomics 2014;15:162. https://doi.org/10.1186/14712164-15-162

30.Drummond AJ, Suchard MA, Xie D, Rambaut A. Bayesian Phylogenetics with BEAUti and the BEAST 1.7. Mol Biol Evol 2012;29:1969-73. https://doi.org/10.1093/molbev/mss075

31. Browning BL, Browning SR. A unified approach to genotype imputation and haplotype-phase inference for large data sets of trios and unrelated individuals. Am J Hum Genet 2009;84: 210-23. https://doi.org/10.1016/j.ajhg.2009.01.005

32.Pfeifer B, Wittelsbürger U, Ramos-Onsins SE, Lercher MJ. PopGenome: an efficient Swiss army knife for population genomic analyses in R. Mol Biol Evol 2014;31:1929-36. https:// doi.org/10.1093/molbev/msu136

33. Huang DW, Sherman BT, Lempicki RA. Systematic and integrative analysis of large gene lists using DAVID bioinformatics resources. Nat Protoc 2009;4:44-57. https://doi.org/10.1038/ nprot.2008.211

34. Reiner A, Yekutieli D, Benjamini Y. Identifying differentially expressed genes using false discovery rate controlling procedures. Bioinformatics 2003;19:368-75. https://doi.org/10.1093/ bioinformatics/btf877

35.Davis TL, Walker JR, Campagna-Slater V, et al. Structural and biochemical characterization of the human cyclophilin family of peptidyl-prolyl isomerases. PLoS Biol 2010;8:e1000439. https://doi.org/10.1371/journal.pbio.1000439

36. Prins BP, Mead TJ, Brody JA, et al. Exome-chip meta-analysis identifies novel loci associated with cardiac conduction, including ADAMTS6. Genome Biol 2018;19:87. https://doi.org/10. 1186/s13059-018-1457-6

37. Park AC, Phan N, Massoudi D, et al. Deficits in col5a2 expression result in novel skin and adipose abnormalities and pre- disposition to aortic aneurysms and dissections. Am J Pathol 2017;187:2300-11. https://doi.org/10.1016/j.ajpath.2017.06. 006

38. Cheeseman IM, Hori T, Fukagawa T, Desai A. KNL1 and the CENP-H/I/K complex coordinately direct kinetochore assembly in vertebrates. Mol Biol Cell 2008;19:587-94. https:/doi. org/10.1091/mbc.e07-10-1051

39. Burbach BJ, Medeiros RB, Mueller KL, Shimizu Y. T-cell receptor signaling to integrins. Immunol Rev 2007;218:65-81. https://doi.org/10.1111/j.1600-065X.2007.00527.x

40.Zhao B, Tumaneng K, Guan KL. The hippo pathway in organ size control, tissue regeneration and stem cell self-renewal. Nat Cell Biol 2011;13:877-83. https://doi.org/10.1038/ncb2303

41. Khanal RC, Nemere I. Regulation of intestinal calcium transport. Ann Rev Nutr 2008;28:179-96. https://doi.org/10.1146/ annurev.nutr.010308.161202

42. Aksanov O, Green P, Birk RZ. BBS4 directly affects proliferation and differentiation of adipocytes. Cell Mol Life Sci 2014; 71:3381-92. https://doi.org/10.1007/s00018-014-1571-x

43. Wang Q, Ning H, Peng $H$, et al. Tristetraprolin inhibits macrophage IL-27-induced activation of antitumour cytotoxic T cell responses. Nat Commun 2017;8:867. https://doi.org/10. 1038/s41467-017-00892-y

44. Richard AC, Peters JE, Savinykh N, et al. Reduced monocyte and macrophage TNFSF15/TL1A expression is associated with susceptibility to inflammatory bowel disease. PLoS Genet 2018;14:e1007458. https://doi.org/10.1371/journal.pgen.1007 458

45. Koochakzadeh L, Hosseinverdi S, Hedayat M, et al. Study of SH2D1A gene mutation in paediatric patients with B-cell lymphoma. Allergol Immunopathol 2015;43:568-70. https:// doi.org/10.1016/j.aller.2015.01.007

46. Fischl H, Howe FS, Furger AM, Mellor J. Paf1 has distinct roles in transcription elongation and differential transcript fate. Mol Cell 2017;65:685-98. https://doi.org/10.1016/j.molcel. 2017.01.006

47. Wang X, Song Q. Mst1 regulates post-infarction cardiac injury through the JNK-Drp1-mitochondrial fission pathway. Cell Mol Biol Lett 2018;23:21. https://doi.org/10.1186/s11658-0180085-1

48. Glavey SV, Manier S, Natoni A, et al. The sialyltransferase ST3GAL6 influences homing and survival in multiple myeloma. Blood 2014;124:1765-76. https://doi.org/10.1182/blood-201403-560862

49. Horiuchi N, Kumagai D, Matsumoto K, Inokuma H, Furuoka $\mathrm{H}$, Kobayshi Y. Detection of the nonsense mutation of OPA3 gene in Holstein Friesian cattle with dilated cardiomyopathy in japan. J Vet Med Sci 2015;77:1281-3. https://doi.org/10. 1292/jvms.15-0150

50.Nishi A, Numata S, Tajima A, et al. De novo non-synonymous TBL1XR1 mutation alters Wnt signaling activity. Sci Rep 2017; 7:2887. https://doi.org/10.1038/s41598-017-02792-z 